\title{
DEDUZIONE DELLE CONCENTRAZIONI DI URANIO E TORIO NELLE SOSTANZE RADIOATTIVE CON IL METODO FOTOGRAFICO
}

\author{
G. Imbò - L. Casertano
}

1. - Nella nota precerlente ( $\left.{ }^{1}\right)$ si è data la distribuzione statistica delle particelle a raccolte su una lastra fotografica, in funzione della proiezione sulla lastra del percorso della particella nelI'emulsione; tale distribuzione $\dot{c}$ stata studiata sia per le particelle a emesse da una sola specie di nucleo radioattivo, sia per quelle provenienti da tutti gli elementi di ogni singrola famiglia in equilibrio radioattivo.

Ora prima di dare - come si era detto - le distribuzioni per le particelle emesse dall'insieme delle tre famiglie, e in funzione anclue del rapporto in peso della concentrazione dell'uranio $\left(C_{1}\right)$ rispetto a quella del torio $\left(\mathrm{C}_{\mathrm{ru}}\right)$ in modo da poter ricavare questo rapporto dalle distribuzioni che si ottengono in pratica, si è voluto riesaminare il problema trattato per un completamento dal punto di vista analitico, tenendo presente, a tale scopo, i lavori successivamente pubblicati sull'argomento.

Quasi contemporaneamente al nostro lavoro, difatti, Buttlar (*) e Hautermans ( ${ }^{2}$ ) hanno trattato lo stesso prolulema presso a poco nellidentico modo scguito da noi: unica differenza sostanziale è che essi per evitare la soluzione analitica in it della già riferita equazione:

$$
\underline{r}=R \operatorname{sen} \theta-\frac{x}{\operatorname{tg} t}
$$

(dlove $r$ i la proiezione del percorso nell'enulsione di una particella $\alpha$ emessa sotto l'angolo it da un volume elementare posto a distanza $x ; R$ il percorso nell'aria; $\mu_{x}$ il coeficiente di frenamento della sostanza radioattiva e $\mu_{\text {r }}$ quello dell'enulsionej e quindi dell'integrale che permette di ricavare il totale delle particelle is rac-

(*) $\mathrm{Si}$ è vivantente grati al dott. Butrusk perché ci ha inviato copia cicIostilata delIa sua tesi. 
colte con proiezione maggiore di $r$, ricorrono alle soluzioni grafiche; mentre noi preferimmo - come esposto - trovare la distribuzione statistica delle particelle $a$ emesse da uno strato di spessore $x={ }_{11} R$ come limite delle distribuzioni di un numero sempre crescente di strati infinitamente sottili posti a distanze comprese fra 0 e $\mu_{1} R$.

I risultati ottenuti nei due modi non potevano non concordare: in particolare $\mathrm{i}$ valori dati precedentemente nella Tabella III per le tre famiglie radioattive in equilibrio confrontati con quelli delle loro Tabelle IIa, IIb, IIc presentano al massimo un scostamento intorno all' $1 \%$.

Più recentemente si è occupato ancora dell'argomento il Van Stijvendael $\left({ }^{3}\right)$ che, impostando il problema in modo solo formalmente diverso, è arrivato a una formula analoga a quella data dall'Evans $\left({ }^{4}\right)$ per le lunghezze effettive dei percorsi nell'emulsione anziché delle proiezioni.

Tale formula però può ovviamente essere ricavata anche partendo dallimpostazione data da noi e da Buttlar e Hautermans.

2. - Tenendo conto di quanto detto nella precedente nota si può facilmente vedere come il numero $d n$ di particelle " emesse dal volume elementare $d S d x$ posto alla distanza $x$ dall'emulsione, raccolte sulla lastra e aventi proiezione maggiore di $r$, essendo $\frac{1-\cos i}{2}$ l'angolo solido corrispondente a iे, è dato da:

$$
d n=\frac{N}{2}\left(\cos \hat{\vartheta}_{z}-\cos \vartheta_{1}\right) d S d x
$$

dove $\cos \vartheta_{1}$ e $\cos \hat{v}$, sono radici della [1] per i considerati valori di $x$ e $r$.

Poiché la [1] può anche porsi sotto la forma:

$$
x=u_{1} k \cos \vartheta-r \frac{\mu_{1}}{\mu_{0}} \frac{\cos \vartheta}{\operatorname{sen} \vartheta}
$$

si può ottenere, per $r$ costante:

$$
d x=\mu_{1} R\left(\frac{r}{\mu_{2} R \operatorname{sen}^{2} \vartheta}-\operatorname{sen} \vartheta\right) d \vartheta
$$

Con facili considerazioni (v. fig. 1 ) si ricava che il numero $n$ di particelle $\alpha$ emesse da uno strato di spessore $x$, raccolte sulla lastra 


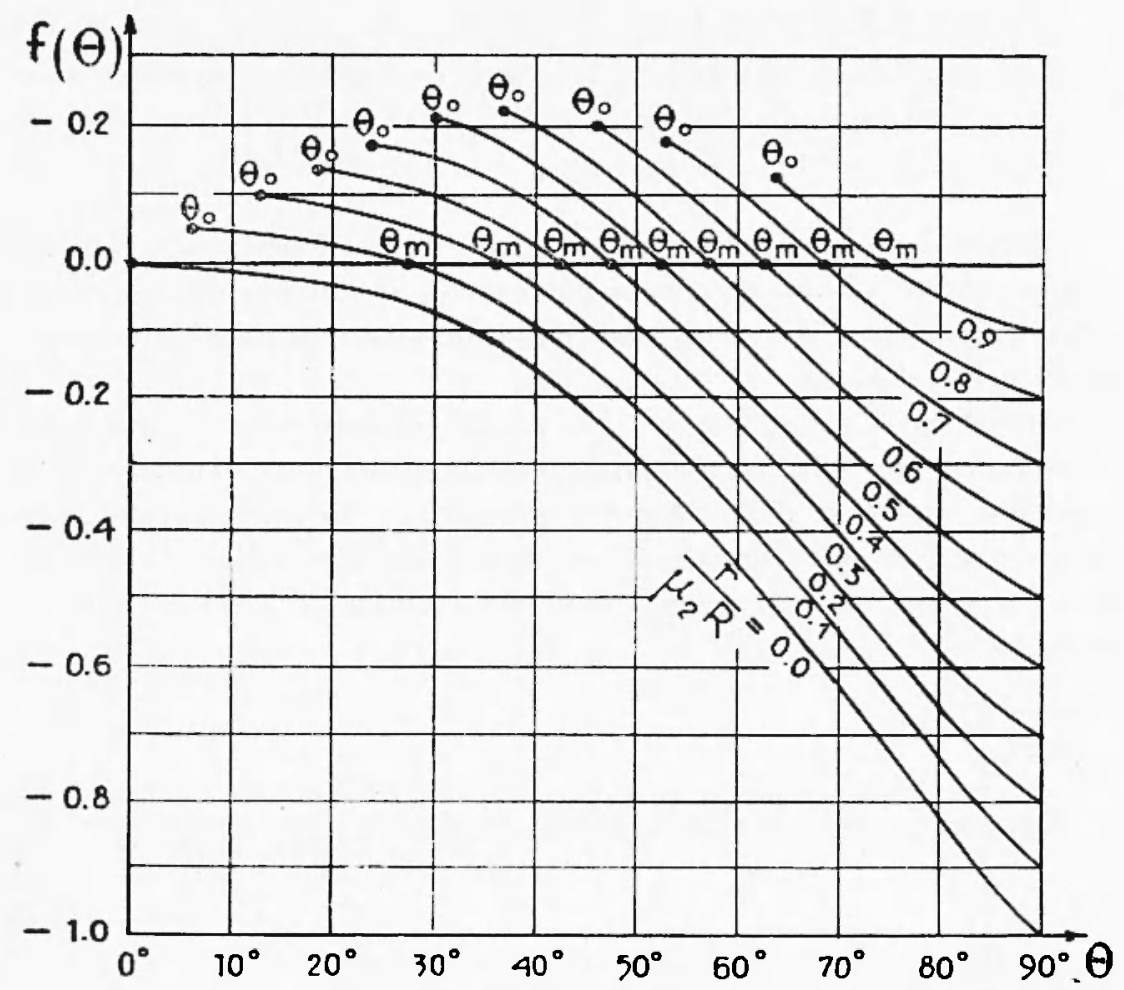

Fig. 1 - Variazione di $f(\Theta)$ (ordinate) in funzione di $\Theta$ (ascisse) per valori discreti di $r / \mu_{2} R$.

per unità di superficie e aventi proiezioni del percorso nell'emulsione maggiore di $r$ è dato da:

$$
n=\frac{N}{2}\left[\int_{\vartheta_{1}}^{\vartheta_{0}} f(\vartheta) d \vartheta-\int_{\vartheta_{2}}^{\vartheta_{0}} f(\vartheta) d \vartheta\right]
$$

dove si è posto $f(\vartheta)=(1-\cos \vartheta)\left(\frac{r}{\mu_{2} R \operatorname{sen}^{2} \vartheta}-\operatorname{sen} i\right) j$ e le coppie $\left(\vartheta_{0} \vartheta_{0}^{\prime}\right)$ e $\left(\vartheta_{1} \vartheta_{2}\right)$ sono radici della [l] in corrispondenza del posto valore di $r$ per le distanze dei due piani che limitano lo strato considerato. In particolare poiché si suppone la sostanza a contatto con l'emulsione si avrà sempre:

$$
\vartheta_{0}=\operatorname{arcsen} \frac{r}{\mu_{2} R} \quad \text { e } \quad \vartheta_{0}^{\prime}=\frac{\pi}{2}
$$


Quando si considera $x=\mu_{1} R$ si avrà :

$$
n=\frac{N}{2} \int_{\vartheta_{0}^{\prime}}^{\vartheta_{0}} f(\vartheta) d \vartheta=\frac{N}{4} \mu_{1} R\left(1-\frac{r}{\mu_{2} R}\right)^{2}
$$

Questo è da considerarsi un caso limite. Infatti per $x>u_{1} R$ la [5] risulta sempre valida, perché la parte della sostanza a distanza maggiore di $\mu_{1} R$ non dà alcun contributo in quanto $\mathrm{i}$ raggi non penetrano nell'emulsione.

Invece per $x<\underline{u}_{4} R$ la [5] ̀̀ valida soltanto se si considerano proiezioni $r$ maggiori della proiezione massima corrispondente alla superficie superiore dello strato di spessore $x$. In generale per spessori $x<\mu_{i} R$ le distribuzioni si debbono ricavare dalla [4] giovandosi dei valori $\vartheta_{1}$ e $\vartheta_{g}$ deducibili con facilità dal grafico riportato in fig. 2.

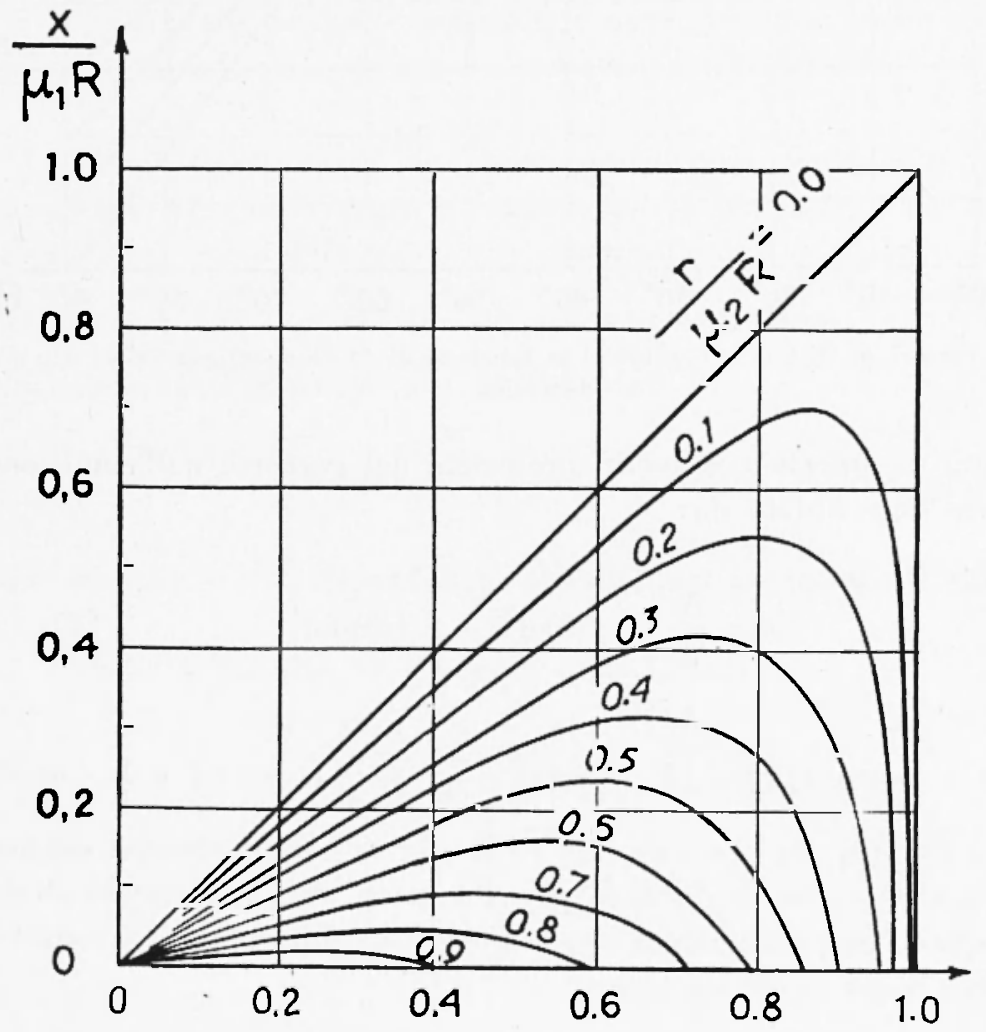

Fig. 2 - Variazione di $\cos \Theta$ (ascisse) in funzione $\mathrm{di} x / \mu_{t} R$ (ordinate) per valori discreti di $r / \mu_{2} R$. 
Nella Tabella I sono date alcune distribuzioni. In tale tabella, tra parentesi, su ogni colonna è riportato il totale di particelle $\alpha$ raccolte sulla lastra per i vari spessori (ciò̀ il numero di particelle $r$ con proiezione maggiore di zero): si è posto uguale a 100 quello relativo allo spessore $x=\mu_{1} R$.

\section{TABELLA I}

\begin{tabular}{|c|c|c|c|c|c|c|}
\hline$U_{n} R$ & $\begin{array}{r}0.0 \\
(0.0)\end{array}$ & $\begin{array}{r}0.2 \\
(36)\end{array}$ & $\begin{array}{r}0.4 \\
(64)\end{array}$ & $\begin{array}{r}0.6 \\
(84)\end{array}$ & $\begin{array}{r}0.8 \\
(96)\end{array}$ & $\begin{array}{c}1.0 \\
(100)\end{array}$ \\
\hline 0.0 & 0.0 & 100.0 & 100.0 & 100.0 & 100.0 & 100.0 \\
\hline 0.2 & 0.0 & 92.8 & 96.3 & 76.2 & 66.7 & 64.0 \\
\hline 0.4 & 0.0 & 68.6 & 56.3 & 42.9 & 37.5 & 36.0 \\
\hline 0.6 & 0.0 & 44.4 & 25.0 & 19.0 & 16.7 & 16.0 \\
\hline 0.8 & 0.0 & 11.1 & 6.3 & 4.8 & 4.2 & 4.0 \\
\hline 1.0 & 0.0 & 0.0 & 0.0 & 0.0 & 0.0 & 0.0 \\
\hline
\end{tabular}

Distribuzioni statistiche delle particelle $r$, in funzione della proiezione $(r /, R)$ sulla lastra del percorso nell'emulsione per diversi valori dello spessore $\left(x / u_{1} R\right)$ dello strato della sostanza emittente.

Qualche apparente anomalia della variazione della percentuale delle particelle $a$ con proiezione maggiore di un determinato valore, in funzione dello spessore, trova la piena spiegazione nel comportamento - accennato sopra - dipendente dalla validità o meno della [5], per $x<\mu_{1} R$, a seconda che $r$ è maggiore o minore della $r$ massima, caratteristica dello spessore considerato.

Altro spessore limite può essere considerato quello infinitamente sottile (invece di quello con $x=0$ ) e cioè quello per il quale si ritiene che tutti i nuclei emittenti siano a contatto con l'emulsione.

Di questo facilmente se ne puó trovare la distribuzione. Se $N^{\prime}$ è il numero di particelle $\alpha$ emesse per secondo e per grammo di sostanza, la frazione $(p)$, rispetto al totale di particelle emesse, con proiezione minore di $r$ è data da:

$$
p=N^{\prime} \frac{1-\cos \vartheta}{2}
$$

Essendo $\frac{N^{\prime}}{2}$ il numero totale delle particelle $a$ raccolte per grammo di sostanza, $d$ la densità della sostanza, e $\cos i)=\sqrt[l]{1-\left(\frac{r}{u_{2} R}\right)^{2}}$, il na 
mero $n$ di particelle $\&$ raccolte per $\mathrm{cm}^{2}$ di superficie e con proiezione maggiore di $r$ sarà:

$$
n=\frac{N d}{2} \sqrt{1-\left(\frac{r}{\mu_{2} R}\right)^{2}}
$$

In pratica sono prevalentemente considerati solamente $i$ due spessori limiti, e cioè quello infinitamente sottile e l'altro per cui si ha $x=\mu_{1} R$ : in quanto al primo vi si ricorre per particolari esigenze e al secondo per la facilità con la quale esso può essere ottenuto (basta che si abbia $x>\mu_{1} R$. Si osserva che mentre sul numero totale di tracce si ha nel primo caso un valore inferiore che nel secondo (al quale corrisponde il massimo): sulle distribuzioni invece si ha per lo spessore infinitamente sottile un netto predominio della percentuale di percorsi a più grandi proiezioni.

Per queste ragioni per l'insieme delle famiglie radioattive vengono prese in considerazione solo distribuzioni che si hanno per questi due spessori. Mentre però per l'infinitamente sottile il campo di variazione delle proiezioni sarà esteso da $r=0$ a $r=\mu_{2} R$, per $x={ }_{11} R$ sarà limitato da $0,60<r<\mu_{2} R$, in quanto si sono volute, in questo secondo caso, trascurare le più piccole proiezioni.

3. - Per le famiglie in equilibrio radioattivo, indicando con $N^{*}$ il numero di particelle ${ }^{t}$ emesse al secondo e per gr di sostanza da ognuno degli elementi in equilibrio, e con $d$ la densita della sostanza, le [5] e [6] diventano rispettivamente:

e

$$
n=\frac{\Lambda^{*}}{4} \mu_{1} d \Sigma_{\mathrm{i}}\left(1-\frac{r}{\mu_{2} R_{\mathrm{i}}}\right)^{2}
$$

$$
n=\frac{N^{*} d}{2} \Sigma_{\mathrm{i}}{\sqrt{1-\left(\frac{r}{\mu_{2} R_{\mathrm{i}}}\right)^{2}}}^{2}
$$

con le sommatorie estese a tutti quegli elementi della famiglia il cui percorso nell'aria, $R_{1}$, è maggiore dell'equivalente in aria, $\frac{r}{l_{2}}$, della
proiezione $r$.

Essendo i capostipiti delle famiglie dell'uranio e dell'attinio due isotopi dello stesso elemento chimico (rispettivamente $U_{238}$ e $U_{235}$ ) l'insieme delle due famiglie sarà considerato come una famiglia unica (uranio-attinio) con due sostanze madri, cui corrispondono evidentemente due valori diversi di $N^{*}$. In particolare per i due valori di $N^{*}$ si sa che un grammo di uranio $(U I+U I I+A c-U) \dot{e}$ con- 
posto da $0,9928 \mathrm{gr}$ di $U I\left(U_{238}\right)$ e di $0,0072 \mathrm{gr}$ di $A c-U\left(U_{235}\right)$, essendo trascurabile il contributo dell'UII $\left(U_{234}\right)$. Perciò in base alle costanti di disintegrazione può ritenersi che 1 gr di uranio, in condizione di equilibrio, emette al secondo 24986 particelle $\alpha$, delle quali 12210 provenienti da ognuno dei due isotopi $U_{33 s}$ e $U_{231}$, e 566 provenienti dal terzo $U_{235}$.

Per la famiglia del torio invece è da considerare che dal rapporto di ramificazione del $T h C$ si ricava che di 100 particelle $a$ provenienti dalla disintegrazione del $T h C, 35$ sono emesse dal $T h C$ e 65 dal $T h C^{\prime}$. Per il numero di particelle $\alpha$ emesse al secondo e per gr di sostanza per ogni elemento in equilibrio della famiglia del torio si ricava il valore di 4100 .

TABELLA II

\begin{tabular}{|c|c|c|c|c|c|c|c|c|c|}
\hline \multicolumn{5}{|c|}{$x_{2} 0$} & \multicolumn{5}{|c|}{$x=u_{1} R$} \\
\hline \multirow[b]{2}{*}{$\frac{r}{\mu_{0} K}$} & \multicolumn{2}{|c|}{ Uranio-Altinio } & \multicolumn{2}{|c|}{ Torio } & \multirow[b]{2}{*}{$\frac{r}{\mu_{2} K}$} & \multicolumn{2}{|c|}{ Úranio-Attinio } & \multicolumn{2}{|c|}{ Torio } \\
\hline & $\frac{N}{u}$ g p.g & $\%$ & $\begin{array}{c}\frac{N}{d} \mathrm{~g} \mathrm{p.g} \\
\text { di } \mathrm{Th}\end{array}$ & $\%$ & & $\begin{array}{l}\frac{N}{u_{1}} \bar{a} \mathrm{p} . \\
\mathrm{g} d i U\end{array}$ & $\%$ & $\frac{N}{\mu_{1} d}$ g & $\%$ \\
\hline 0.0 & 50821 & 100.0 & 12300 & 100.0 & 0.60 & 1428 & 100.0 & 959 & 100.0 \\
\hline 0.1 & 49308 & 97.0 & 12023 & 97.7 & 0.64 & 966 & 67.7 & 744 & 77.6 \\
\hline 0.2 & 44458 & 87.5 & 11129 & 90.5 & 0.68 & 526 & 36.9 & 585 & 61.0 \\
\hline 0.3 & 34196 & 67.3 & 8844 & 71.9 & 0.72 & 243 & 17.1 & 448 & 46.7 \\
\hline 0.4 & 16126 & 31.7 & 7122 & 57.9 & 0.76 & 70 & 4.9 & 329 & 34.3 \\
\hline 0.5 & 7837 & 15.4 & 3967 & 32.3 & 0.80 & 1 & 0.9 & 228 & 23.8 \\
\hline 0.6 & 4425 & 8.7 & 1933 & 15.7 & 0.84 & 0 & 0.0 & 146 & 15.2 \\
\hline 0.7 & 3146 & 6.2 & 951 & 7.7 & 0.88 & 0 & 0.0 & 82 & 8.6 \\
\hline 0.8 & 385 & 0.8 & 799 & 6.5 & 0.92 & 0 & 0.0 & 37 & 3.8 \\
\hline 0.9 & 0 & 0.0 & 580 & 4.7 & 0.96 & 0 & 0.0 & 9 & 1.0 \\
\hline 1.0 & 0 & 0.0 & C & 0.0 & 1.00 & 0 & 0.0 & 0 & 0.0 \\
\hline
\end{tabular}

Distribuzioni statistiche per le famiglie radioattive in equilibrio. Sia gli spessori $(x)$ della sostanza che le proiezioni $(r)$ del percorso nell'emulsione sono state riferite al persorso nell'aria del $\mathrm{ThC}^{\prime}(\mathrm{R}=\mathrm{cm} 8.57)$ che $\dot{\mathrm{e}}$ il massimo per le partirelle ".

Nella Tabella II si riportano le distribuzioni ricavate: i valori di $\frac{r}{\mu_{2}}$, per $\mathrm{i}$ successivi confronti, sono stati sempre riferiti al massimo percorso per le particelle a (uguale a $\mathrm{cm} 8,57$ per $T h C^{\prime}$ ).

5. - Dalle colonne 2 e 4 per $x>\hat{u}$ : e da quelle 7 e 9 per $x=:_{t} R$ si possono ricavare le distribuzioni per qualsiasi determinato rap- 
porto $C_{\mathrm{v}} / C_{\mathrm{Th}}$ delle concentrazioni in gr per gr dell'uranio $\left(C_{\mathrm{t}}\right)$ rispetto a quelle, sempre in gr per gr, del torio $\left(C_{\mathrm{rh}}\right)$.

Infatti, per $x \simeq 0$, la colonna 2 dà senz'altro la distribuzione per $C_{\mathrm{V}} / C_{\mathrm{Th}}=\infty$ e la 4 quella per $C_{\mathrm{V}} / C_{\mathrm{rh}}=0$. Mentre dalla somma delle due colonne si può ottenere l'altra per $C_{\mathrm{V}} / C_{\mathrm{Th}}=1$; e così via. $\mathrm{E}$ analogamente può dirsi per lo spessore $x=\mu_{\mathrm{l}} R$.

Nelle Tabelle III e IV sono riportati i valori ottenuti.

TabelLA III

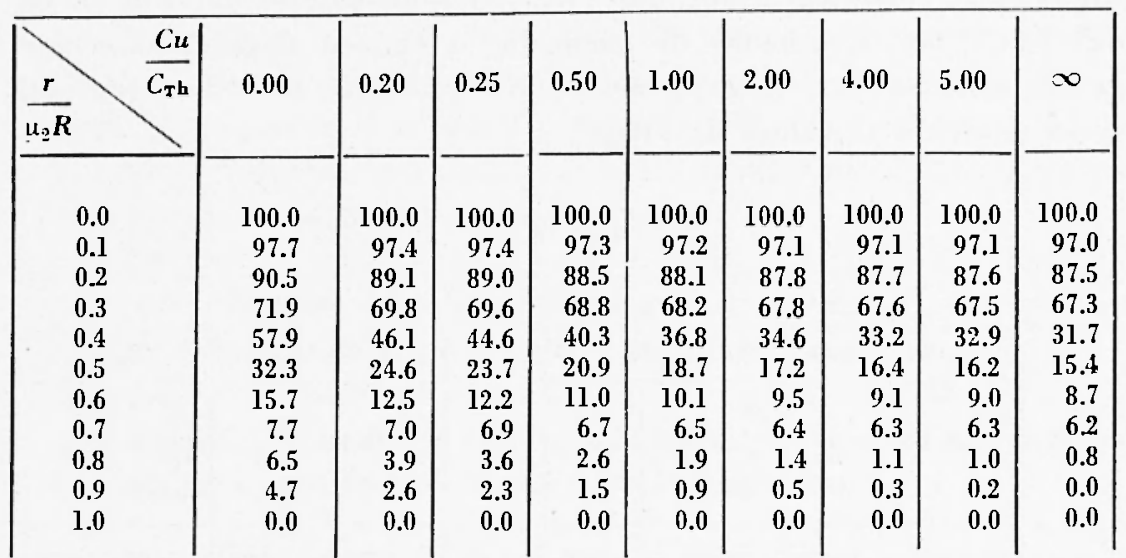

Distribuzioni statistiche per diversi valori del rapporto $\mathrm{C}_{\mathrm{u}} / \mathrm{C}_{\mathrm{Th}}$ per lo spessore infinitamente sottile della sostanza. Il valore di $r / !_{2} R$ è riferito al percorso nell'aria del ThC' $(R=\mathrm{cm} 8,57)$.

TABella IV

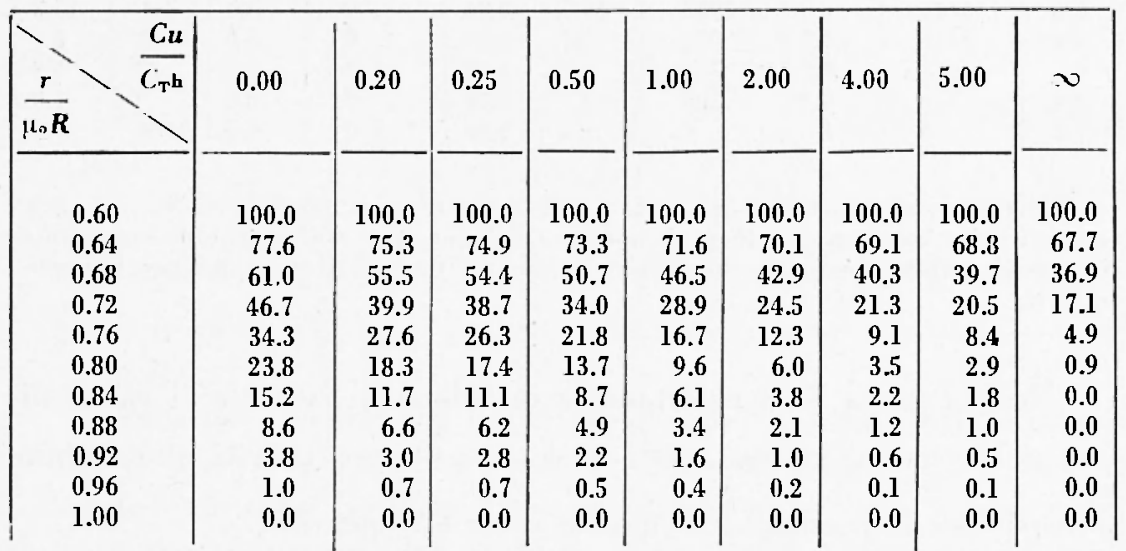

Distribuzioni statistiche per diversi valori del rapporto $C_{u} / C_{T h}$ per lo spessore $x=\mu_{1} R$ della sostanza. Il valore di $r / \mu_{2} R$ è riferito al percorso nell'aria del ThC $\mu(\mathrm{R}=\mathrm{cm} 8,57)$. 
Per poter calcolare il rapporto $C_{\mathrm{v}} / C_{\mathrm{Th}}$; nei grafici delle figg. 3 e 4 sono riportati - rispettivamente per $x \geq \hat{v}$ e per $x=u_{i} R-$ gli andamenti, per valori discreti di $\frac{r}{\mu_{1} R}$, delle variazioni delle percentuali (ascisse) con il variare del rapporto in esame (ordinate). Riportando su ogni curva il valore della percentuale ottenuta sperimentalmente può calcolarsi facilmente il valore del rapporto, ricorrendo eventual-

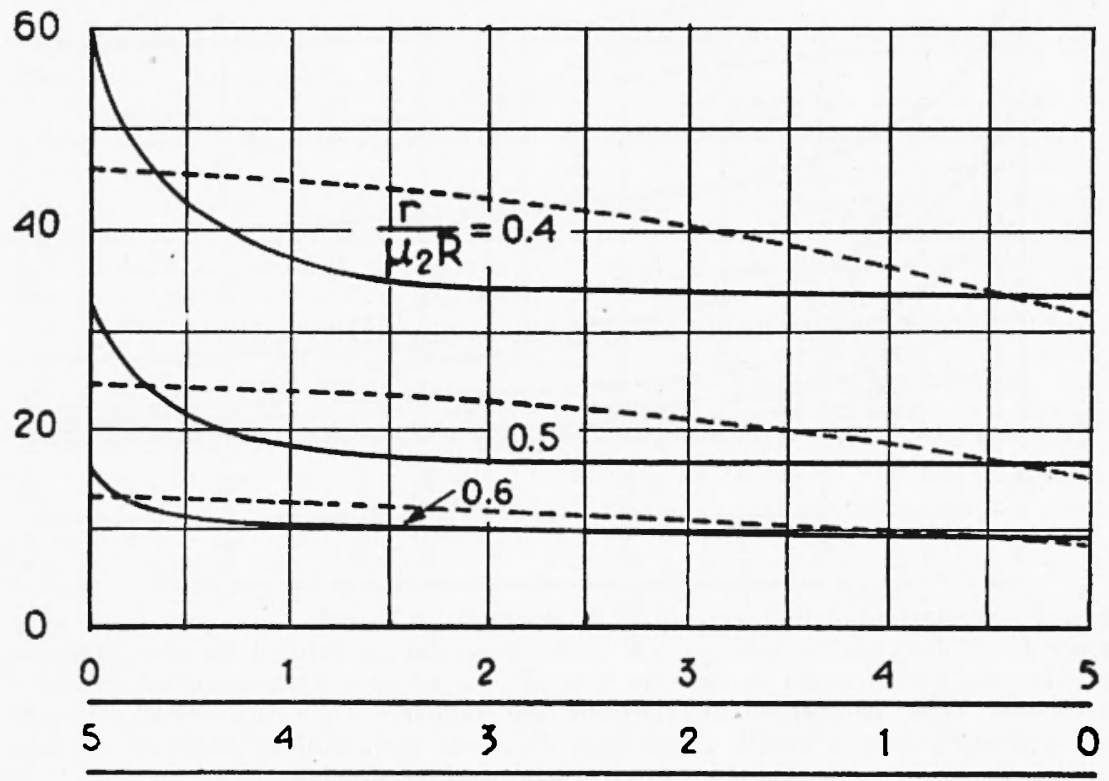

Fig. 3 - Variazioni delle percentuali di particelle $\alpha$ raccolte con proiezioni maggiori di un determinato valore $r / \mu_{2} R$ in funzione del rapporto delle concentrazioni uranio-torio per lo strato infinitamente sottile. Le curve a tratto continuo danno le variazioni delle percentuali in funzione del rapporto $\mathrm{Co}_{\mathrm{o}} / \mathrm{C}_{\mathrm{Th}}$ (ascisse crescenti verso destra); mentre quelle a tratteggio danno le variazioni in funzione del rapporto $\mathrm{C}_{\mathrm{Th}} / \mathrm{C}_{u}$ (ascisse crescenti verso sinistra).

mente anche a una media ponderata dei valori ricavati per diverse proiezioni.

Per rendere illimitato il campo di variazione del rapporto $C_{\text {. }} / C_{\tau \mathrm{th}}$ nei grafici sono riportati anche gli andamenti del rapporto inverso $C_{\mathrm{Th}} / C_{\mathrm{v}}$; in tal modo si può scegliere tra $\mathrm{i}$ due rapporti quello che più conviene prendere in considerazione. 


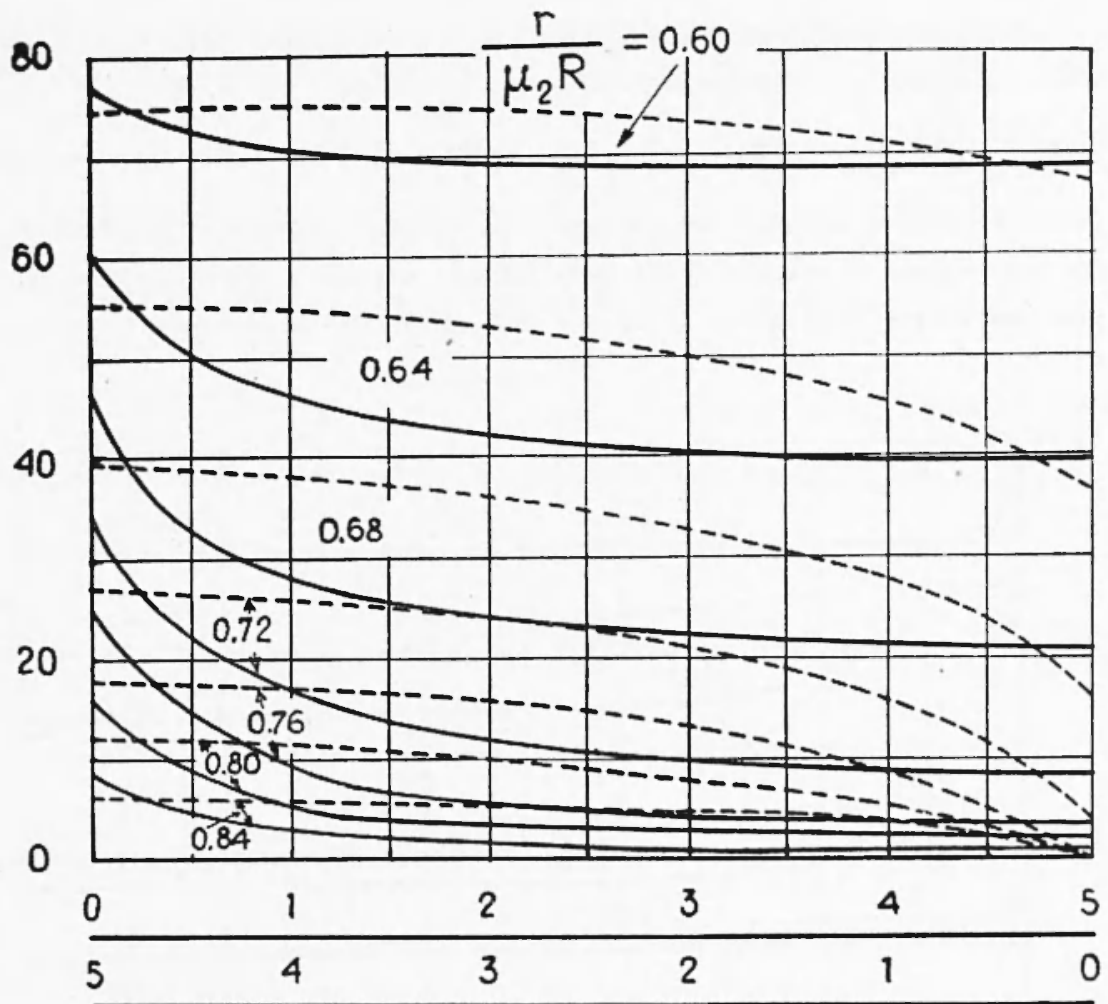

Fig. 4 - Variazioni delle percentuali di particelle a raccolte con proiezioni maggiori di un determinato valore $r / \mu . R$ in funzione del rapporto delle concentrazioni uranio-torio per lo strato di spessore $\mathrm{x}=\mu_{1} \mathrm{R}$. Le curve a tratto continuo danno le variazioni delle percentuali in funzione del rapporto $\mathrm{C}_{6} ; \mathrm{C}_{\mathrm{T}}$ h (ascisse crescenti verso destra); mentre quelle a tratteggio danno le variazioni in funzione del rapporto $\mathrm{C}_{\mathrm{rh}} / \mathrm{C}_{\mathrm{V}}$ (ascisse crescenti verso sinistra).

I grafici delle figg. 5 e 6 permettono di ricavare, in funzione del rapporto calcolato distintamente, il numero di particelle u raccolte per secondo e per unità di superficie relativamente allo strato infinitamente sottile nonché il numero di particelle la cui proiezionc equivalente in aria risulta maggiore $\mathrm{di} \mathrm{cm} \mathbf{5 , 1 4 2}$ (per lo strato di spes. sore $x=u_{1} R$.

Quindi il numero effettivamente raccolto, per unità di superficie e per secondo, di particelle $a$ con proiezione analoga a quella considerata per ognuno dei due strati, permette di ricavare anche le singole concentrazioni degli elementi uranio e torio. Infatti i calcoli sono stati condotti ammettendo:

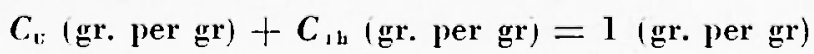




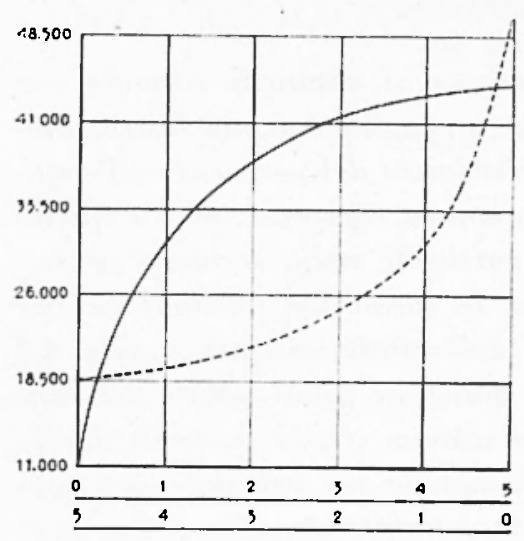

Fig. 5 - Variazione di $\mathrm{N} / \mathrm{d}$ ( $\mathrm{N}$ numero totale di particelle \& raccolte per secondo e per unitì di superficie, d densità della sostanza) in funzione del rapporto delle concentrazioni uranio-torio per lo strato infinitamente sottile. La curva a tratto continuo dà la variazione $\mathrm{di} N / \mathrm{d}$ in funzione del rapporto $C_{r} / C_{\text {th }}$ (ascisse crescenti verso destra); mentre quella a tratteggio dà la variazione in funzione del rapporto $\mathrm{C}^{1 /} / \mathrm{C}_{\mathrm{u}}$ (ascisse crescenti verso sinistra).

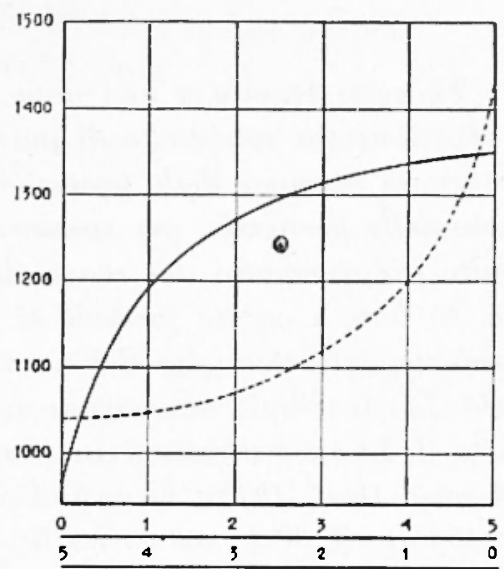

Fig. 6 - Variazione di $N / \|_{1} d(N$ numero di particelle $\alpha$ raccolte per secondo e per unità di superficie con proiezione $r / u_{2} R \geqslant 0,60 ; \quad u_{1}$ coefficiente di frenamento e d densità della sostanza) in funzione del rapporto delle concentrazioni urano-torio per lo strato di spessore $x=u_{1} R$. La curva a tratto continuo dà la variazione di $\mathrm{N} / \mathrm{H}_{1} \mathrm{~d}$ in funzione del rapporto $\mathrm{C}_{\mathrm{V}} / C_{\mathrm{r}}$ (ascisse crescenti verso destra); mentre quella a tratteggio dà la variazione in funzione del rapporto $C_{h} / C_{c}$ (ascisse crescenti verso sinistra).

Ora se $\dot{e}$ stato trovato $C_{\mathrm{l}} / C_{\mathrm{Th}}=p$ e si pone uguale a $q$ il rapporto tra il totale di particelle a raccolte e quello che si sarebbe ricavato se fosse effettivamente valida la [7] si ottiene facilmente:

$$
C_{:=}=\frac{p q}{1+p} \quad \text { e } \quad C_{\text {m. }}=\frac{q}{1+p}
$$

Basandosi pertanto sull'ipotesi dell'esistenza dell'equilibrio radioattivo con questa nota si è voluto indicare il metodo che si può seguire per conoscere, dalle distribuzioni statistiche delle parlicelle $\iota$, in funzione della proiezione sulla lastra del percorso nell'emulsione, il valore non solo del rapporto ma anche delle singole concentrazioni dell'uranio e del torio relative alle sostanze esaminate.

\section{Napoli - Osservatorio Vesuviano - Dicembre 1951.}




\section{RIASSUNTO}

Facendo seguito a una nota precedente si danno le formule per le distribuzioni statistiche di particelle a raccolte, su una lastra fotografica, in funzione della proiezione sulla lastra del percorso nell'emulsione delle particelle, per spessori di sostanza compresi fra 0 e quello limite rappresentato dal range delle particelle nella sostanza in esame. In base a queste formule si danno le stesse distribuzioni sia per l'insieme delle famiglie dell'uranio e dell'attinio, sia per quella del torio. Le distribuzioni vengono anche date per fissati valori del rapporto delle concentrazioni in peso delluranio $\left(\mathrm{C}_{v}\right)$ rispetto a quelle del torio $\left(\mathrm{C}_{\mathrm{Th}}\right)$. Infine viene indicato il metodo per calcolare non solo il rapporto $\mathrm{C}_{\mathrm{v}} / \mathrm{C}_{\mathrm{Th}}$ ma anche le singole concentrazioni $\mathrm{C}_{\mathrm{n}}$ e $\mathrm{C}_{\mathrm{th}}$ partendo dalle distribuzioni statistiche, ottenute sperimentalmente, in funzione delle proiezioni sulla lastra dei percorsi nell'emulsione delle particelle a emesse dalla sostanza esaminata.

\section{SUMMARY}

In pursuance of a previous note, formulae are here given to obtain the statistical distribution of a particles as gathered on photographic plates, in function of the projection on the plate of the particle trajectory inside of the emulsion, for thickness values varying from zero to the limit thickness represented by the particle range in the substance under examination. Starting from these formulae, distributions have been indicated both for the whole group of Uranium and Actinium, and for the Thorium group. Distributions have been also indicated for fixed ratio values of Uranium weight concentrations $\left(\mathrm{C}_{\mathrm{c}}\right)$ in respect of Thorium weight concentrations $\left(\mathrm{C}_{T \mathrm{~h}}\right)$. Further has been described the method used for the calculation not only of rations $\mathrm{C}_{\mathrm{v}} / \mathrm{C}_{\mathrm{Th}}$, but also of the single concentrations $\mathrm{C}_{\mathrm{a}}$, and $\mathrm{C}_{\mathrm{Th}}$, as experimentally deduced from the projections on the plate of the trajectories in the emulsion of the a particles wich are emitted by the substance under examination.

\section{BIBLIOGR AFIA}

(1) Imbò e Casfrtano: Ann. Geof. Vol, IIl, 475, 1950.

(2) Buttlar: Tesi di laurea; Buttlar e Hautermans: Geoc. el Cosm. Ac. Vol. II, 43, 1951 .

(3) Van Stijvendael: Bul. Cen. Phys. Nucl. N. 28, 1951.

(4) Evans: Phys. Rev., 45, 29, 1934. 